\title{
High Level of Heat Shock Protein 25 (Hsp25) Expression in Atherosclerotic Lesions
}

\section{Govindasamy llangovan}

Davis Heart and Lung Research Institute and Division of Cardiovascular Medicine, Wesxner Medical Center, The Ohio State University, Columbus, OH 43082, USA

"Corresponding author: Govindasamy llangovan, Davis Heart and Lung Research Institute and Division of Cardiovascular Medicine, Wesxner Medical Center, The Ohio State University, Columbus, OH 43082, USA, Tel: 6142929064; E-mail: Govindasamy.ilangovan@osumc.edu

Rec date: Nov 02, 2014, Acc date: Nov 04, 2014, Pub date: Nov 06, 2014

Copyright: ( 2014 llangovan G. This is an open-access article distributed under the terms of the Creative Commons Attribution License, which permits unrestricted use, distribution, and reproduction in any medium, provided the original author and source are credited.

\section{Description}

Confocal microscopic analysis of Immunofluroscence of triple stained aortic atherosclerotic lesions in low density lipids receptor knock out (LDLr-/-) mouse. Purpose of this study was to analyze a small heat shock protein, namely Hsp25, expression in atherosclerotic lesions. LDL-/- mice were fed with high cholesterol containing western diet to create aortic lesions. Descending aorta was isolated from these mice and stained with florescent dye tagged antibodies: GREEN channel actin; BLUE channel DAPI; and RED channel Hsp25. Actin in intima is clearly seen without overlap with red color. However in lipid rich lesions, yellow colored blocks are observed, as indication these smooth muscle layers containing the lipid cores. The yellow blocks indicate the high in Hsp25, in addition to high red fluorescence likely from not cellular structure and perhaps the lipid rich area. This is key evidence showing high Hsp25 expression in atherosclerotic lesions (Figure 1).

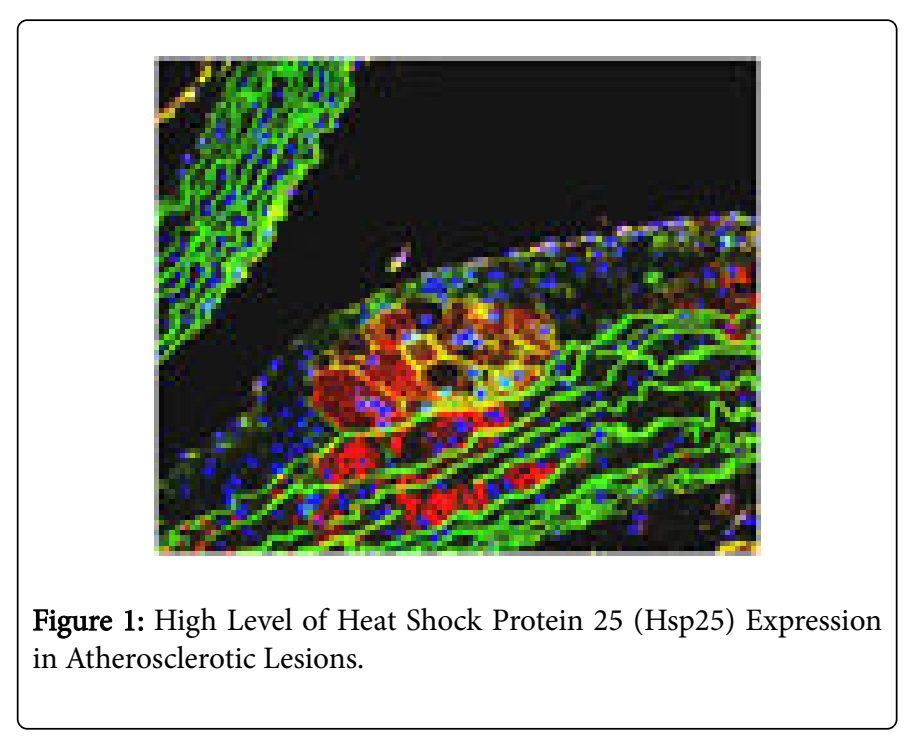

